\title{
Tailoring the surgical corridor to the basilar apex in the pretemporal transcavernous approach: morphometric analyses of different neurovascular mobilization maneuvers.
}

\author{
Timothy G White ${ }^{1} \cdot$ Giyarpuram N Prashant $^{1,2} \cdot$ Prashin Unadkat $^{1} \cdot$ Amir R Dehdashti $^{1}$
}

Received: 6 July 2020 / Accepted: 10 July 2020 / Published online: 18 July 2020

(C) Springer-Verlag GmbH Austria, part of Springer Nature 2020

We commend the authors for their efforts to formalize an approach to basilar apex aneurysms. Key to the approach of any aneurysm is the establishment of proximal control as well as the ability to dissect and visualize the neck of the aneurysm to ensure appropriate clip placement, which can be particularly challenging in basilar aneurysms given its anatomic location and deep perforators. Given the variable anatomy of the basilar artery, numerous approaches and schema have been developed and described in the literature [1]. Historically, the pterional and subtemporal approaches were described to access the basilar apex; however, numerous other techniques have been developed to facilitate access to the basilar artery and expand the surgical corridor, including the orbitozygomatic craniotomy, various transpetrosal approaches, and the endoscopic transclival approach [1-6].

This paper provides a systematic approach to classifying the surgical corridors and techniques that can be used to enhance access to the basilar artery from a frontotemporal approach. It describes access to the basilar artery through three common corridors: the opticocarotid (OCT), the carotid oculomotor (COT), and the oculo-tentorial (OTT). Baseline measurements of these surgical windows were calculated in cadaveric specimens after completing an orbitozygomatic craniotomy. Subsequently, specific maneuvers were used to expand access, including division of the carotid distal dural ring, opening of the oculomotor triangle, mobilization of the

This article is part of the Topical Collection on Vascular Neurosurgery Other

Amir R Dehdashti

adehdashti@northwell.edu

1 Department of Neurosurgery, Donald and Barbara Zucker School of Medicine at Hofstra Northwell, Hempstead, NY, USA

2 Department of Neurosurgery, University of California, Los Angeles, CA, USA oculomotor nerve, and posterior clinoidectomy. Through this method, "normal" dimensions of each of these windows were compared before and after neurovascular mobilization. The included figures provide an excellent illustration of these methods and the exposure gained.

While we appreciate the effort to study this approach and provide quantitative measurements of specific corridors, it is important to note that in many cases, the approach will be dictated largely by the patient's individual anatomy and intraoperative findings. The surgical windows described in this manuscript must be explored intraoperatively to determine the optimal trajectory and often are used in tandem, for instance a basilar artery proximal control through OTT and aneurysm exposure through COT. The addition of a posterior clinoidectomy is generally dictated by height of the basilar apex, which can be evaluated on pre-operative imaging. Therefore, we believe that while it is helpful to have an understanding of the normal dimensions of these corridors and the maneuvers that can be used to expand visualization, careful examination of the pre-operative films combined with thorough exploration intraoperatively are ultimately necessary for successful outcomes in these challenging cases regardless of the provided dimensions.

The manuscript details the advantages various surgical corridors have on the exposure of the basilar artery. Maneuvers such as mobilization of the ICA (increases higher basilar access above the dorsal sella) by opening of the carotid cuff, mobilization of the oculomotor nerve, and drilling of the posterior clinoid help improve exposure. In our opinion, with use of adjuvant techniques, the COT allows for an overall greater access to the basilar artery, making it the most versatile corridor. On the other hand, the OTT allows for a more complete lower access, especially of the proximal basilar control without the need for any further mobilization or dissection. The OCT allows for the highest access to the basilar when used alone without any adjuvant techniques; however, it is most often limited and have less range of motion compared with 
COT. Drilling off the posterior clinoid in all cases significantly increases access of the proximal basilar artery, but not always needed. Ultimately, drilling the anterior clinoid process can give further lateral exposure by mobilization of carotid artery along with transcavernous exposure and opening of the oculomotor canal. This will allow a wider exposure of the basilar artery complex and access to proximal basilar lateral to the oculomotor nerve. The latter is the approach we use for more complex basilar top aneurysms for which the widest possible exposure is indicated.

Probably, the most impactful finding of the paper is the described impact of drilling the posterior clinoid process providing experimental evidence that drilling of this process enhances basilar access. However, this will certainly vary patient to patient. While the differences in exposure cited here were significant, the range (for example $\pm 2.5 \mathrm{~mm}$ after drilling the posterior clinoid process accessing through the COT) was high. Indeed, these measurements, albeit a nice systematic evaluation, will likely not significantly impact our surgical approach as they just confirm what is already well established based on expert opinion [7].

\section{References}

1. Tayebi Meybodi A, Benet A, Rodriguez Rubio R, Yousef S, Lawton MT (2018) Comprehensive anatomic assessment of the pterional, orbitopterional, and orbitozygomatic approaches for basilar apex aneurysm clipping. Oper Neurosurg (Hagerstown) 15(5):538-550

2. Dolenc VV, Škrap M, Šušteršič J, Škrbec M, Morina A (1987) A transcavernous-transsellar approach to the basilar tip aneurysms. Br J Neurosurg 1(2):251-259

3. Kawase T, Toya S, Shiobara R, Mine T (1985) Transpetrosal approach for aneurysms of the lower basilar artery. J Neurosurg 63(6): 857-861

4. Seoane E, Tedeschi H, de Oliveira E, Wen HT, Rhoton AL Jr (2000) The pretemporal transcavernous approach to the interpeduncular and prepontine cisterns: microsurgical anatomy and technique application. Neurosurgery 46(4):891-899

5. Somanna S, Babu RA, Srinivas D, Rao KVN, Vazhayil V (2015) Extended endoscopic endonasal transclival clipping of posterior circulation aneurysms - an alternative to the transcranial approach. Acta Neurochir 157(12):2077-2085

6. Tayebi Meybodi A, Benet A, Rodriguez Rubio R, Yousef S, Mokhtari P, Preul MC et al (2018) Comparative analysis of orbitozygomatic and subtemporal approaches to the basilar apex: a cadaveric study. World Neurosurg 119:e607-ee16

7. González LF, Amin-Hanjani S, Bambakidis NC, Spetzler RF (2005) Skull base approaches to the basilar artery. Neurosurg Focus 19(2): $1-12$

Publisher's note Springer Nature remains neutral with regard to jurisdictional claims in published maps and institutional affiliations. 